\title{
Neurocognitive improvements following endovascular repair of vein of Galen malformation in a child
}

\author{
Robyn A. Howarth, PhD, ${ }^{1,4}$ Andrew Reisner, MD,,3,4 Joshua J. Chern, MD, PhD, 2,4 \\ Laura L. Hayes, MD, ${ }^{4}$ Thomas G. Burns, PsyD, ${ }^{1,3,4}$ and Alejandro Berenstein, MD ${ }^{5}$ \\ Departments of ${ }^{1}$ Neuropsychology, ${ }^{2}$ Neurosurgery, and ${ }^{3}$ Pediatrics, Emory University School of Medicine, Atlanta; ${ }^{4}$ Children's \\ Healthcare of Atlanta, Georgia; and ${ }^{5}$ Ichan School of Medicine at Mount Sinai Health Systems, New York, New York
}

\begin{abstract}
Cognitive regression is a well-described presentation of vein of Galen aneurysmal malformations (VGAMs) in childhood. However, it remains unclear whether successful treatment of the malformation can reverse cognitive regression. Here, the authors present the case of a 5 -year-old girl with a VGAM that was treated with staged endovascular embolization procedures. Comprehensive neurocognitive assessments were completed before intervention and approximately 6 years after initial presentation. There were significant age-matched improvements in this child's neurocognitive profile over this period. The authors believe that timely and successful treatment of VGAM in children may not only stabilize the associated cognitive deterioration but, in some cases, may ameliorate these deficits. Details of this case and a discussion of neurocognitive deficits related to VGAM are presented.
\end{abstract}

http://thejns.org/doi/abs/10.3171/2014.10.PEDS14244

KEY WORDS vein of Galen; endovascular procedures; neuropsychological tests; pediatric neurosurgery; vascular disorders

$\mathrm{V}$ EIN of Galen aneurysmal malformations (VGAMs) classically present with cardiac failure and/or hydrocephalus in neonates and infants. ${ }^{16}$ In rare cases, VGAMs occur later in childhood, usually with cognitive deficits ${ }^{16,17,19}$ Although the pathophysiology of this worsening cognition is not fully understood, venous hypertension with resulting venous ischemia is a major contributor that, if left untreated, may progress to global cerebral ischemia as well as a "melting-brain" syndrome. ${ }^{16,17}$ Other etiological factors of this decline include congenital neuronal abnormalities associated with the VGAM, acquired progressive subcortical ischemia, and/or other unidentified factors. Radiographic markers of this phenomenon have been well described and include progressive widespread subcortical calcifications, venous hypertension, and cerebral atrophy. ${ }^{17,22}$ The indications for intervention once subcortical calcifications are present remain controversial, and whether successful occlusion of the malformation can reverse cognitive regression has not been reported previously to our knowledge. Here, we report a case of a 5-yearold, right-handed girl with a VGAM who was treated with staged endovascular embolizations, resulting in total oc- clusion of the malformation. Comprehensive neurocognitive assessments were completed before intervention and at approximately 6 years after presentation. There were significant age-matched improvements in this child's neurocognitive profile over this period. We believe that timely and successful treatment of VGAMs in children may not only stabilize the associated cognitive deterioration but, in some cases, may result in reversal of these deficits.

\section{Case Report}

History

The patient was born full term weighing approximately 7 pounds, and no complications with the pregnancy or birth were reported. Developmental milestones generally were reached within age-typical expectations, and the patient initially demonstrated adequate academic development during her early preschool years. The parents began to notice gradual increasing difficulty with aspects of early learning between ages 4 and 5 years. The patient received early intervention services in the school setting before being diagnosed with VGAM. Early medical history was sig- 
nificant for mild reactive airway disease, which was well controlled with occasional inhaler therapy.

\section{Examination}

The patient initially presented to Pediatric Neurosurgery Associates at age 5.3 years because of a several-month history of progressively worsening cognitive dysfunction, which the parents had attributed initially to problematic behavior. For example, the patient displayed new difficulty with self-care activities such as dressing herself. In addition, despite previously being aware of her surroundings, she would become disoriented on the school bus, not understanding why she was there. An initial head CT scan completed at an outside facility showed signs of VGAM (Fig. 1). In addition, evidence of widespread dystrophic calcifications was revealed in the frontal and temporoparietal regions. This calcification primarily occurred in the subcortical white matter. Additional rim calcifications were seen involving the wall of VGAM. Initial CT findings prompted further neurological evaluation.

On examination, the patient's head circumference was $50 \mathrm{~cm}$, which placed her in the 60th percentile for her age. Craniofacial features were normal, and there was no cranial bruit. A brief bedside evaluation revealed higher functions that were age appropriate. Cranial nerve and neurological examinations were normal, with no focal deficits or signs of intracranial hypertension.

After the revelation of VGAM, with extensive subcortical dystrophic calcifications, the following tests were recommended: MRI (Fig. 2) with MR angiography, MR venography, and neuropsychological testing. Further surgical or endovascular intervention would be explored based on the results of these studies.

\section{Presurgical Neuropsychological Assessment}

Initial neuropsychological testing was completed at age 5.5 years, after diagnosis and before intervention. The battery of neuropsychological assessments included measures of intellectual functioning, verbal and visual memory, language-processing skills, visuomotor skills, and academic achievement that have been standardized and normed with large representative samples. Scores were standardized by
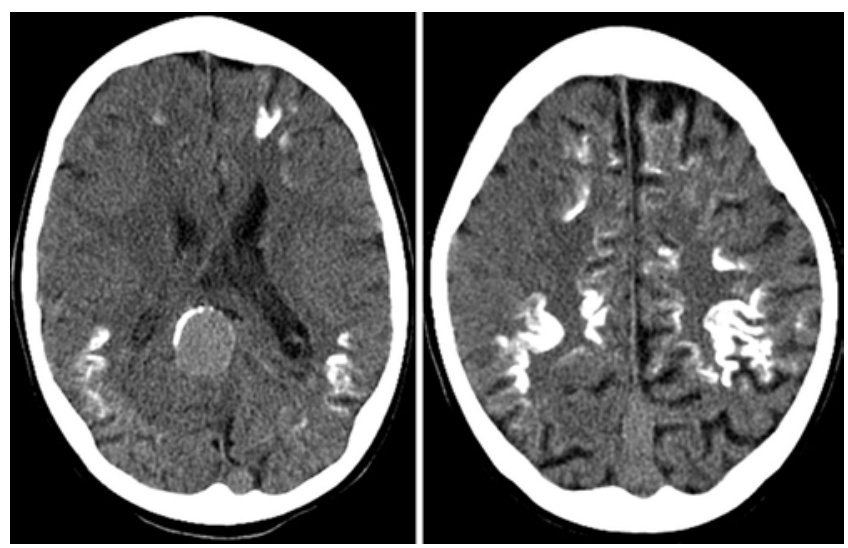

FIG. 1. Preembolization CT scans of the head. Noncontrast scans demonstrate a hyperdense VGAM with rim calcifications. Note the extensive dystrophic calcifications in the subcortical white matter with mild, diffuse volume loss.
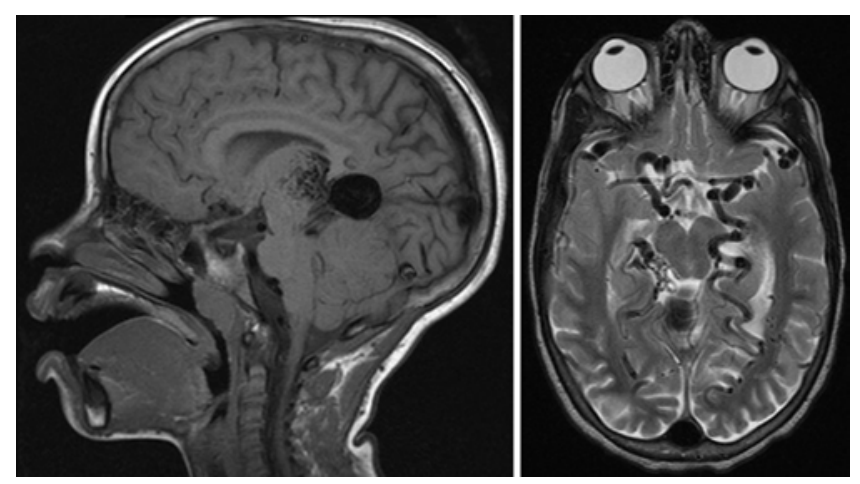

FIG. 2. Initial MR images of the brain. Left: Sagittal, noncontrast, T1weighted image demonstrating the flow voids in the VGAM, with deep arteriovenous malformation nidus, draining into a falcine dural channel. Right: Axial T2-weighted image revealing dilated, tortuous arterial branches of the anterior circulation.

age, with a mean of 100 and a standard deviation of 15 . The parents also completed two questionnaires (Behavior Rating Inventory of Executive Function, Behavior Assessment System for Children, Second Edition) that examined the real-world manifestation of various executive functions as well as aspects of social-emotional and behavioral functioning in children.

At the time of initial assessment, findings revealed overall intellectual functioning in the extremely low range; however, there was a significant discrepancy noted between the patient's low average verbal reasoning compared with less well-developed nonverbal reasoning skills (Table 1). Specific areas of weakness included aspects of visual-spatial processing, fine motor output (e.g., fine motor speed, motor coordination, copying activities), and emerging executive functions (e.g., attention, cognitive shifting, problem solving). Comprehension difficulties were also noted, although expressive language skills were largely intact. The parents' responses revealed significant concerns with aspects of executive and social-emotional functioning in the everyday environment. Significant concerns were also reported for anxiety, withdrawal, and atypical behaviors.

\section{Endovascular Intervention}

A 5-stage endovascular embolization process over 3 years, using flow-guided microcatheter techniques and $N$-butyl 2 cyanoacrylate (Trufill, Codman), was needed to completely obliterate the malformation and relieve the venous congestion (preembolization angiogram-Fig. 3A and $\mathrm{B}$; postembolization angiogram-Fig. $3 \mathrm{C}$ and D).

\section{Postembolization Neuropsychological Assessment}

Follow-up neuropsychological testing was completed approximately 18 months after the patient's most recent embolization procedure when she was age 11.1 years. Compared with the initial assessment, updated findings suggested significant improvements in her neurocognitive profile compared with age-matched norms, as indicated by relative stability or improvement of standard scores (Table 1; Fig. 4). It is noteworthy that the presence of higher standard scores on the posttreatment assessment suggests not only sustained learning over time but also ac- 
TABLE 1. Neuropsychological data

\begin{tabular}{|c|c|c|c|}
\hline \multirow[b]{2}{*}{ Test } & \multicolumn{2}{|c|}{ Standard Scores* } & \multirow[b]{2}{*}{ Performance Change } \\
\hline & Pre-Tx (age 5-6 yrs) & Post-Tx (age 11.1 yrs) & \\
\hline \multicolumn{4}{|l|}{ Intellectual functioning } \\
\hline $\mathrm{VIQ} / \mathrm{VCI}$ & 81 & 95 & $\ddagger$ \\
\hline PIQ/PRI & 51 & 61 & $\ddagger$ \\
\hline \multicolumn{4}{|l|}{ Verbal memory } \\
\hline \multicolumn{4}{|l|}{ Verbal learning } \\
\hline Immediate & 85 & 95 & $\ddagger$ \\
\hline Delay & 80 & 100 & $\dagger$ \\
\hline Recognition & 85 & 120 & $\dagger$ \\
\hline \multicolumn{4}{|l|}{ Story memory } \\
\hline Immediate & 110 & 100 & \\
\hline Delay & 85 & 105 & $\dagger$ \\
\hline Recognition & 95 & 105 & $\ddagger$ \\
\hline \multicolumn{4}{|l|}{ Visual memory } \\
\hline \multicolumn{4}{|l|}{ Picture memory } \\
\hline Immediate & 100 & 65 & \\
\hline Delay & 115 & 110 & \\
\hline \multicolumn{4}{|l|}{ Language } \\
\hline Comprehension & 60 & 80 & $\dagger$ \\
\hline Receptive vocabulary & 82 & 98 & $\dagger$ \\
\hline Expressive vocabulary & 80 & 102 & $\dagger$ \\
\hline \multicolumn{4}{|l|}{ Visual perception/visuomotor } \\
\hline Visual motor integration & 66 & 52 & \\
\hline Visual perception & 63 & 77 & $\ddagger$ \\
\hline \multicolumn{4}{|l|}{ Fine motor speed } \\
\hline \multicolumn{4}{|l|}{ Pegs } \\
\hline Dominant hand (right) & 53 & 76 & $\dagger$ \\
\hline Nondominant hand (left) & 70 & 63 & \\
\hline \multicolumn{4}{|l|}{ Academic achievement } \\
\hline Word reading & 90 & 88 & \\
\hline Calculation & 60 & 65 & \\
\hline
\end{tabular}

$\mathrm{PIQ} / \mathrm{PRI}=$ Performance Intelligence Quotient/Perceptual Reasoning Index; $\mathrm{Tx}=$ treatment; $\mathrm{VIQ} / \mathrm{VCI}=$ Verbal Intelligence Quotient/Verbal Comprehension Index.

* Neuropsychological data are presented as standard scores, with a mean of 100 and SD of 15.

$\dagger$ Improvement was conceptualized as significant for performance that improved by at least 1 SD compared with peers of same age.

$\ddagger$ Performance that improved by less than 1 SD was conceptualized as trending toward improvement.

celerated improvement compared with peers of the same age. For the purpose of this single-case design, significant improvement was conceptualized as performance that improved by at least $1 \mathrm{SD}$ ( $>15$ points). Performance that improved $<1 \mathrm{SD}$ was conceptualized as trending toward improvement. Verbal reasoning, language processing, and verbal memory skills were largely age appropriate as opposed to below average performance across visual-spatial tasks (e.g., visual organization, visual matching, visual memory). Significant improvements were noted for both verbal and nonverbal tasks, including measures of word knowledge, comprehension, verbal memory, visual matching, and dominant (right-)hand fine motor speed. Attention was also much improved during testing compared with the previous evaluation. Parent responses further endorsed improved attention and executive functioning in the every- day environment. Stable and improved scores suggested that the patient continued to make gains at the same rate or faster than her age-matched peers in these areas; however, immediate visual memory performance was notably lower than the prior assessment.

\section{Discussion}

A VGAM represents a rare vascular anomaly characterized by arteriovenous fistulas and shunts that drain into a dilated median vein of Markowski., $1,6,24$ The lesion is located in the midline and often receives blood supply from the limbic system, including bilateral choroidal arcades, pericallosal arteries, variable transmesencephalic arteries, and perforators of the midbrain. ${ }^{18}$ The etiology of VGAMs is unclear, although arteriovenous shunts are 


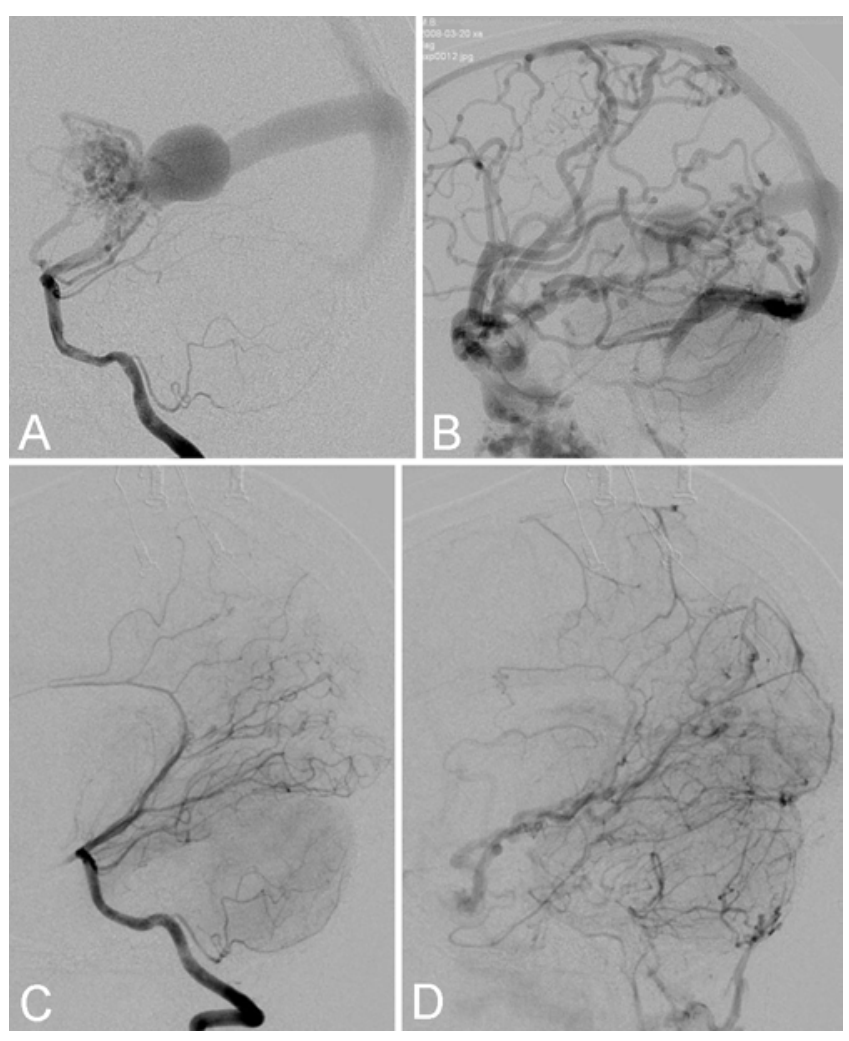

FIG. 3. Vertebral artery cerebral angiograms. Lateral views in the arterial $(\mathrm{A})$ and venous $(\mathrm{B})$ phases prior to treatment demonstrate the deep arteriovenous malformation with early filling of the aneurysmal vein of Galen and falcine channel draining into the superior sagittal sinus. Note the prominent veins draining preferentially into the cavernous sinuses and pterygoid plexus. Lateral views in the arterial (C) and venous (D) phases following the third embolization procedure demonstrate complete obliteration of the malformation and resolution of venous congestion.

thought to develop early in fetal development, between the 6th and 11th weeks of gestation..$^{25}$ Raybaud and Strother ${ }^{24}$ and Raybaud et al..$^{25}$ confirmed that the vein involved with VGAMs is the embryonic precursor of the vein of Galen. VGAM has since been recognized as an embryonic choroid plexus vascular malformation..$^{18}$

The clinical presentation of VGAMs depends on a variety of factors, including the age of the child; the size and location of the lesion; the status of venous drainage such as outflow restrictions or thrombosis, which can redirect venous outflow of the brain and/or the malformation through collateral pathways such as the cavernous sinus; and/or transosseous veins. ${ }^{16,17,22,23}$ Specifically, the presence of venous hypertension can progress to cortical calcifications or even a so-called melting-brain syndrome, which represents an irreversible consequence. ${ }^{16,17}$ Neonates typically present with signs of significant cardiorespiratory distress and/or high-output cardiac failure. ${ }^{26}$ Infants often present with macrocephaly, hydrocephalus, and/or seizures. ${ }^{11,13}$ Some infants have been found to present with developmental delay or failure to thrive associated with complicating medical factors (e.g., long-standing cerebral venous hypertension, cardiac decompensation). ${ }^{4,13}$ Other signs and symptoms may include cranial bruit, dilated scalp veins, proptosis, and/or recurrent epistaxis. ${ }^{11}$ Although rare, older children and adults tend to present with relatively more innocuous symptoms, such as headache, signs associated with subarachnoid hemorrhage, or learning difficulties. , $11,13^{2}$

Early reports of VGAM were associated with a poor prognosis and high mortality rates..$^{12}$ The development of endovascular techniques, including recent advances in embolization procedures, has led to a notable improvement in survival and mortality rates. ${ }^{3,5,7,9,21}$

\section{Cognitive Outcome in VGAM}

Reports of cognitive impairment and regression have been documented in the context of VGAM, which is often accompanied by radiographic markers of progressive widespread subcortical calcifications and cerebral atrophy. ${ }^{17}$ The presence of bilateral and symmetrical cerebral calcifications has been reported previously to coincide with VGAM, and these calcifications are typically located in the subcortical venous watershed areas. ${ }^{6}$ Reports suggest that these calcifications are often associated with brain atrophy and may represent evidence of a venous hypoxic disorder. ${ }^{6}$ Although the presence of calcifications indicates an insult to the developing brain, the calcifications have not been shown to be predictive of neurological outcome..$^{18}$

Despite advancements in treatment approaches, only a limited number of reports have examined the neurocognitive outcomes specifically associated with VGAM. ${ }^{8,10,18,20,27}$ On the basis of retrospective chart review and parent questionnaires, Fullerton et al. ${ }^{10}$ reported that $39 \%$ of surviving patients presented with significant developmental delays, with worse outcomes associated with perinatal presentation, congenital heart failure, and choroidal angioarchitecture. Similar rates of cognitive impairment have been reported by other groups using various assessment measures, including the Bicêtre outcome score. ${ }^{18,20}$ More recently, Ellis et al. ${ }^{8}$ examined the cognitive and functional status of a small cohort of patients $(n=5)$ during the most recent postembolization follow-up appointment (range 2-97 months, median 63 months). A majority of patients $(n=4)$ were reported to be neurologically intact without any cognitive deficits, whereas 1 patient was noted to display premorbid deficits that were unchanged and continued to persist after embolization. It is noteworthy that a majority of outcome studies among pediatric patients with VGAM primarily have used rating scales to determine neurological status rather than performance measures of neurocognitive functioning.

Neurological dysfunction may emerge for a variety of reasons. Because a VGAM forms early in fetal development, cognitive dysfunction may be reflective of alterations in the functional organization and growth of the developing brain, particularly when individuals do not present with a complicating clinical event (e.g., seizures or hemorrhage). Blood flow may be diverted away from the cerebral parenchyma by the large flow of the arteriovenous fistulas, leading to tissue damage and ischemia. ${ }^{14}$ Tissue damage and resulting neurological changes may also result from the presence of associated complications, such as cerebral edema, hypertension, abnormalities in the 


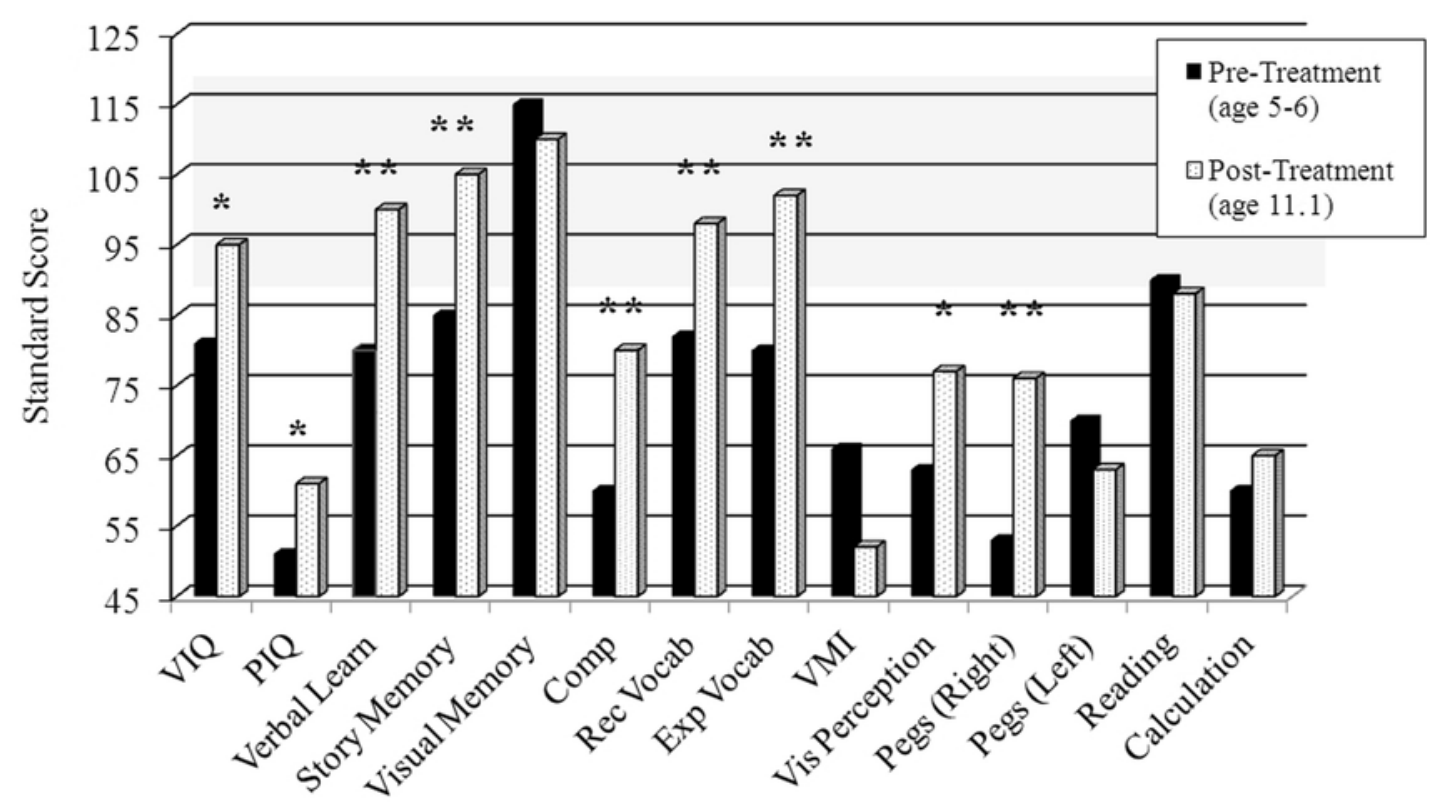

Neuropsychological Assessment Data

FIG. 4. Graph comparing neuropsychological data over time. Neuropsychological data are presented in standard scores, with a mean of 100 and SD of 15. The shaded area (SS 85-115) designates the "average range." Improvement was conceptualized as significant (double asterisks) for performance that improved by at least 1 SD compared with peers of the same age. Performance that improved by $<1$ SD was conceptualized as trending toward improvement (single asterisk). Comp = comprehension; Exp Vocab = expressive vocabulary; Learn = learning; $\mathrm{PIQ}=$ Performance Intelligence Quotient; Rec Vocab = receptive vocabulary; $\mathrm{VIQ}=$ Verbal Intelligence Quotient; Vis = visual; VMI = visual motor integration.

venous system, venous stenosis, and/or thrombosis. All of these factors may further compromise neural tissue perfusion..$^{13,15}$

\section{The Present Case}

In the current case, the patient presented with progressive cognitive changes between ages 4 and 5 years. A recent history of learning differences was also reported, and the patient received early intervention services in the school environment, which were initiated before diagnosis and treatment of the VGAM. These services continued during subsequent school years. No other neurological complaints were reported, and the patient was asymptomatic otherwise. Neuroimaging revealed evidence of a VGAM, with widespread calcifications in the frontal and temporoparietal regions, primarily in the subcortical white matter. The patient underwent a comprehensive neuropsychological evaluation before any neurosurgical intervention at age 5.5 years. Findings indicated overall intellectual functioning in the extremely low range; however, the patient performed better on measures of verbal reasoning than on those of nonverbal reasoning. Additional areas of weakness included visual-spatial processing, fine motor output, emerging executive functions, and comprehension. No stimulant medication was given. The patient underwent an updated comprehensive evaluation approximately 18 months after her most recent embolization procedure at age 11.1 years. Updated results continued to demonstrate a similar pattern of strengths and weaknesses, although the patient exhibited notable improvement in a variety of cognitive domains compared with age-matched norms.
Improvements in neurocognitive functioning may be attributable to multiple factors. First, the patient continued to develop, mature, and be exposed to academic instruction over the 6 years between pre- and posttreatment evaluations. Hence, some improvements may be attributed to typical maturational processes associated with childhood. Second, the embolization procedures have improved cerebral blood flow and relieved venous hypertension, which may contribute to improved neuronal function in various parts of the brain.

Although it is likely that extensive subcortical calcifications are radiographic markers of cognitive impairment, a direct causation and radiographic-clinical correlation has yet to be established definitively. It has been reported that the presence of subcortical calcifications is a marker of severe and potentially irreversible cognitive impairment. ${ }^{6,16-18}$ Less is known about the impact of embolization procedures on improving the cognitive functioning in these individuals. Despite notable areas of neurocognitive weakness before the initiation of treatment, the patient in this case report has continued to make gains in her development compared with peers of the same age. Results continued to suggest greater right hemisphere and frontal lobe disruption compared with largely preserved left hemisphere functioning. Nevertheless, improvements were seen on tasks across domains, including measures of language processing, visual-spatial processing, memory, and attention. We speculate that improvements may be associated, to a certain extent, with the embolization procedures. Without treatment, it is possible that further cognitive deterioration would have continued. 
To our knowledge, this is the first report of a child with VGAM undergoing comprehensive neuropsychological evaluations pre- and postembolization. This report suggests that timely and successful treatment of VGAMs in children may not only stabilize the associated cognitive deterioration but, in some cases, may ameliorate these deficits.

\section{References}

1. Alberico RA, Barnes P, Robertson RL, Burrows PE: Helical CT angiography: dynamic cerebrovascular imaging in children. AJNR Am J Neuroradiol 20:328-334, 1999

2. Alvarez H, Garcia Monaco R, Rodesch G, Sachet M, Krings T, Lasjaunias P: Vein of Galen aneurysmal malformations. Neuroimaging Clin N Am 17:189-206, 2007 (Erratum in Neuroimaging Clin N Am 17:xi, 2007)

3. Berenstein A, Fifi JT, Niimi Y, Presti S, Ortiz R, Ghatan S, et al: Vein of Galen malformations in neonates: new management paradigms for improving outcomes. Neurosurgery 70:1207-1214, 2012

4. Berenstein A, Lasjaunias P: Arteriovenous fistulas of the brain, in: Surgical Neuroangiography. Endovascular Treatment of Cerebral Lesions. Berlin: Springer-Verlag, 1992, Vol 4, pp 267-317

5. Berenstein A, Masters LT, Nelson PK, Setton A, Verma R: Transumbilical catheterization of cerebral arteries. Neurosurgery 41:846-850, 1997

6. Berenstein A, Niimi Y, Song JK, Lasjaunias P: Vein of Galen aneurysmal malformation, in Albright AL, Adelson PD, Pollack IF (eds): Principles and Practice of Pediatric Neurosurgery, ed 2. New York: Thieme Medical Publishers, 2008, pp 1014-1028

7. Berenstein A, Ortiz R, Niimi Y, Elijovich L, Fifi J, Madrid $\mathrm{M}$, et al: Endovascular management of arteriovenous malformations and other intracranial arteriovenous shunts in neonates, infants, and children. Childs Nerv Syst 26:1345-1358, 2010

8. Ellis JA, Orr L, Ii PC, Anderson RCE, Feldstein NA, Meyers PM: Cognitive and functional status after vein of Galen aneurysmal malformation endovascular occlusion. World J Radiol 4:83-89, 2012

9. Friedman DM, Verma R, Madrid M, Wisoff JH, Berenstein A: Recent improvement in outcome using transcatheter embolization techniques for neonatal aneurysmal malformations of the vein of Galen. Pediatrics 91:583-586, 1993

10. Fullerton HJ, Aminoff AR, Ferriero DM, Gupta N, Dowd CF: Neurodevelopmental outcome after endovascular treatment of vein of Galen malformations. Neurology 61:1386-1390, 2003

11. Gailloud P, O'Riordan DP, Burger I, Levrier O, Jallo G, Tamargo RJ, et al: Diagnosis and management of vein of Galen aneurysmal malformations. J Perinatol 25:542-551, 2005

12. Gold A, Ransohoff J, Carter S: Vein of Galen malformation. Acta Neurol Scand Suppl 40 (Suppl 11):1-31, 1964

13. Gupta AK, Varma DR: Vein of Galen malformations: review. Neurol India 52:43-53, 2004

14. Hoang S, Choudhri O, Edwards M, Guzman R: Vein of Galen malformation. Neurosurg Focus 27(5):E8, 2009
15. Khullar D, Andeejani AMI, Bulsara KR: Evolution of treatment options for vein of Galen malformations. J Neurosurg Pediatr 6:444-451, 2010

16. Lasjaunias P, ter Brugge KG, Berenstein A: Surgical Neuroangiography: Clinical and Interventional Aspects in Children, ed 2. New York: Springer, 2006, Vol 3

17. Lasjaunias PL: Vascular Diseases in Neonates, Infants, and Children: Interventional Neuroradiology Management. Berlin: Springer, 1997

18. Lasjaunias PL, Chng SM, Sachet M, Alvarez H, Rodesch G, Garcia-Monaco R: The management of vein of Galen aneurysmal malformations. Neurosurgery 59 (5 Suppl 3):S3184-S3-194, 2006

19. Long DM, Seljeskog EL, Chou SN, French LA: Giant arteriovenous malformations of infancy and childhood. J Neurosurg 40:304-312, 1974

20. McSweeney N, Brew S, Bhate S, Cox T, Roebuck DJ, Ganesan V: Management and outcome of vein of Galen malformation. Arch Dis Child 95:903-909, 2010

21. Mickle JP, Quisling RG: The transtorcular embolization of vein of Galen aneurysms. J Neurosurg 64:731-735, 1986

22. Nass R, Kramer E, Molofsky W, Melnick J, de Hollisey M, Madrid M, et al: Perfusion brain scintigraphy studies in infants and children with malformations of the vein of Galen. Childs Nerv Syst 17:519-523, 2001

23. Nass R, Melnick J, Berenstein A: Temperament profiles of children with vein of Galen malformations. J Child Neurol 13:387-390, 1998

24. Raybaud CA, Strother CM: Persisting abnormal embryonic vessels in intracranial arteriovenous malformations. Acta Radiol Suppl 369:136-138, 1986

25. Raybaud CA, Strother CM, Hald JK: Aneurysms of the vein of Galen: embryonic considerations and anatomical features relating to the pathogenesis of the malformation. Neuroradiology 31:109-128, 1989

26. Rodesch G, Hui F, Alvarez H, Tanaka A, Lasjaunias P: Prognosis of antenatally diagnosed vein of Galen aneurysmal malformations. Childs Nerv Syst 10:79-83, 1994

27. Wong KN, DeLuca J, Strax TE: Cognitive dysfunction following arteriovenous malformation of the vein of Galen: a case analysis. Arch Phys Med Rehabil 77:624-627, 1996

\section{Author Contributions}

Conception and design: all authors. Acquisition of data: Reisner, Howarth. Analysis and interpretation of data: Howarth, Chern, Hayes, Burns, Berenstein. Drafting the article: all authors. Critically revising the article: Howarth, Reisner, Chern, Hayes, Burns. Reviewed submitted version of manuscript: Howarth, Reisner, Chern. Approved the final version of the manuscript on behalf of all authors: Reisner. Statistical analysis: Chern, Burns. Administrative/technical/material support: all authors. Study supervision: Howarth, Reisner, Burns.

\section{Correspondence}

Andrew Reisner, Pediatric Neurosurgery Associates, Children's Healthcare of Atlanta, 5455 Meridian Mark Rd., Ste. 540, Atlanta, GA 30342. email: andrew.reisner@choa.org. 